\title{
Simultaneously Failure of Full Functional Devices and Reduced Functional Devices in Zigbee Sensor Network
}

\author{
Amritpal Kaur \\ M-Tech Scholar \\ Deptt of ECE \\ SBSSTC, Ferozepur, Punjab, \\ India
}

\author{
Jaswinder Kaur \\ Asst. Prof. \\ Deptt of ECE \\ SBSSTC, Ferozepur, Punjab, \\ India
}

\author{
Gurjeevan Singh \\ DIC-ECE \\ Deptt of ECE \\ SBSSTC(Polywing), Ferozepur, \\ Punjab, India
}

\begin{abstract}
This work is based on the characteristics of IEEE 802.15.4 protocol stack. A hybrid topology has been design by using three possible combinations of Zigbee network topologies considered in different scenarios to verify the consistency of this communication network. The scenario has been design under the consideration of device failure in hybrid topologies. There are various parameters like throughput, delay, network load and number hops are measured during these scenarios. The result concludes that Star-Tree hybrid topology is better to make an effective network in case of device malfunctioning. The simulation has been done using OPNET Modeler 14.5. Simulation results enumerate the affect of Zigbee network hybrid topologies on the basis of performance factors.
\end{abstract}

\section{KEYWORDS}

Hybrid Network Topology, Zigbee Wireless Sensor Network, Device Failure.

\section{INTRODUCTION}

Wireless sensing units incorporate wireless communications and mobile computing with transducers to convey a sensor platform which is reasonably priced to set up in several applications. In reality, co-locating computational power and radio frequency (RF) communication inside the sensor unit itself is a distinctive attribute of wireless sensing. The progress in science and technology offers miniaturization, speed, intelligence, erudition, and new materials at inferior cost, which result in the growth of various high-performance smart sensing system [1]. Wireless sensor networks (WSNs), thanks to their collaborative low-cost characteristics, can well suit the needs of the future smart grid. Most likely, wireless sensors will be connected to machineries and other apparatus to monitor faulty behavior and failures of the various components forming the smart grid. In this scenario, wireless sensors are expected to be manifold, have a low duty-cycle (i.e., long periods of inactivity due to low frequency automatic metering traffic), and they should require minimum maintenance. As highlighted in and proper sensor network design (e.g., the selection of suitable protocols) and placement of the sensors on the field, along with suitable radio technologies, represent fundamental steps to enable reliable management and monitoring systems [2]. In recent times, Zigbee has established lots of attentions for its several superior features like rich functions in a small size of device, effective data communication facility in short distance, as well as a low power request and execute cost. Due to these excellent features, Zigbee has been far and wide applying in various areas as a good wireless network solution, particularly where wired network service is infeasible. IEEE 802.15.4 was released by the IEEE 802 wireless personal area network (WPAN) group in 2003, while the Zigbee V1.0 and ZigBee2006 based on the IEEE 802.15.4 were released by the Zigbee Alliance. Zigbee network can be configured in star, tree or mesh topology [3]. Topology formation is an important issue in a wireless sensor network. Performance parameters such as energy consumption, network lifetime, data delivery delay, sensor field coverage depend on the network topology [4]. In particular applications, where admittance of human is difficult WSN play an important role to reach in hazardous areas such as battle fields or disaster areas. The hazards in these areas are also physically fragile the sensor nodes. Usually, sensor nodes are designed to be cheap, very small, and use very limited resources. Therefore, they are not considered to be strong enough to defend against a physical impact. This delicate feature of sensor nodes makes a WSN susceptible to the hazards in such areas. In hazardous area, if some of the sensors nodes become damaged and can no longer work properly, the data from these damaged sensor nodes cannot be collect appropriately. Therefore, the damaged nodes should be repaired by other sensor nodes. The damaged sensor nodes could also block the sink node from collecting data because these sensor nodes are not only data sources but also routing nodes for other source nodes [5]. The main problem of WSNs is reliability. WSNs communications are radio-based and sensors are operated with battery power. Energy depletion, hardware failure, communication link errors, malicious attacks, etc are some causes which may lead to WSNs failure [6]. If nodes are failed then temporary or permanent disconnections in network may occur. This failure of few nodes can cause significant changes in the network like topological changes, packets rerouting and it may also lead to re-organization of the network. In [7] the author only analyzes the performance of tree topology in case of node failure. In this paper, a hybrid network design by using the possible combination of three topologies by using multiple Zigbee coordinators and performance parameters show that which of the combination is best to design the Zigbee sensor network. This paper will cover the study of low data rate protocol Zigbee and also analyze the behavior of network topologies in worst conditions of device malfunctioning.

\section{OVERVIEW TO ZIGBEE}

The Zigbee protocol specifies a wireless technology based on the IEEE 802.15.4 standard for wireless personal area networks (WPANs). The 802.15.4 is a standard that defines the Physical and Medium Access Control (MAC) for low power and low data rate wireless networks [8]. An 802.15.4 network can work both in beacon-enabled or in non-beacon enabled mode. In the beacon enable mode, the network is controlled by a coordinator that provides synchronization and all communications take place using either a slotted CSMA or 
a guaranteed time slot allocation. In the non-beacon mode instead, sensors are not required to be synchronized on a time slot basis and access the medium using an unslotted CSMACA algorithm. Since it is far most widely applied techniques [9]. Zigbee is built on top of 802.15.4 specification and provides a definition for two layers of the OSI model: the Application Layer (APL) and the Network Layer (NWL). Zigbee defines two physical devices: full function devices and reduced function devices. Full function devices (FFD), can work in any topology, are capable of being the Network Coordinator and can talk to any other device in the network. FFD can act as data routers within the network. Reduced function devices (RFD) cannot become a network coordinator can only talk to a network coordinator or router and may have a simple (e.g. hardware) implementation. There is one coordinator in each network (Zigbee Coordinator - ZC), and establishes the network. Routers (Zigbee Router - ZR) act as intermediate nodes, relaying data from other devices. End Devices (Zigbee End Devices - ZED) can be lowpower/battery-powered devices. They have sufficient functionality to talk to their parents (either the coordinator or a router) and cannot relay data from other devices [8]. Zigbee MAC layer employs the CSMA-CA mechanism for channel access and provides secure message transmission over single hop through Advanced Encryption Standard (AES-128) cryptographic algorithm. MAC layers defines frame formats for forming a network based on short (16-bit) and long (64bit) addresses. A long address is unique to each physical node and does not change. A short address is assigned to a node when it joins the network. The network layer is responsible for network structure, routing, and security such as encryption, key management, and authentication [10]. The IEEE 802.15.4 working group has projected to support three different network topologies star, tree and mesh. In mesh topology, a node may communicate with any neighbor, the structure being decentralized. A routing protocol may enable multihop communication, using PAN (personal area network) to PAN transmissions at the MAC layer. In stars, the PAN coordinator (a designated FFD) is in the radio range of all other nodes. A node forms a branch of the star and can communicate only with the PAN coordinator. Single hop transmissions are in this case sufficient for communication. In tree, it presents a generalization of the star topology for multihop communication, enabled at the MAC layer. The coordinators (FFDs) of different clusters (stars) form a tree, rooted at the PAN coordinator. Traffic towards or from the PAN coordinator is forwarded by the coordinators [11].

\section{SIMULATION SCENARIO}

Zigbee has been define the three routing schemes i.e. star, tree and mesh but in this paper the performance is analyzed by making three hybrid topologies by using possible combinations of the different routing schemes. So the hybrid topologies used to analyze under different network configurations are star-tree (ST), star-mesh (SM) and meshtree (MT). The hybrid topologies are designed by using two PAN coordinators in office scale network and two different topologies are assigned to these coordinators. The affect of device failure has been analyzed for these new hybrid networks using different network parameters. The main intention of this work is to quantify the performance of hybrid topologies better than the network containing single coordinator.

The simulation of Zigbee network has been done using OPNET 14.5 and presents the preliminary simulation result to illustrate the attributes of hybrid network in case of node failure. The devised system distributes different Zigbee devices in an area (an office network scale) of $(100 \mathrm{~m} \mathrm{x}$ $100 \mathrm{~m})$. The performance of these networks has been examined under different network configuration as shown in Table 1.

Table 1: Simulation Parameters

\begin{tabular}{|c|c|}
\hline Network Scale & $100 \mathrm{~m} * 100 \mathrm{~m}$ \\
\hline Number Of Nodes & 50 \\
\hline Network Type & Mixed \\
\hline Mobility Model & $\begin{array}{c}\text { Random Waypoint (Record } \\
\text { Trajectory) }\end{array}$ \\
\hline Speed of Mobile Nodes & $2 \mathrm{~m} / \mathrm{s}$ \\
\hline Pause Time & $150 \mathrm{~s}$ \\
\hline Simulation Duration & $300 \mathrm{~s}$ \\
\hline
\end{tabular}

There are three networks, in each network there are two ZC. In case of MT network, tree topology assign to one coordinator and mesh topology assigned to another one. In case of ST network, star topology assign to one coordinator and tree topology assigned to second. In case of SM network, mesh topology assign to one coordinator and star topology assigned to another coordinator. Using above parameter three scenarios has been designed. First scenario analyzed the performance of ST network under ZC, ZR and ZED failure at same time. Second scenario analyzed the performance of SM network under ZC, ZED and ZR failure at same instant of time. Third scenario analyzed the performance of MT network under ZC, ZR and ZED failure at similar time.

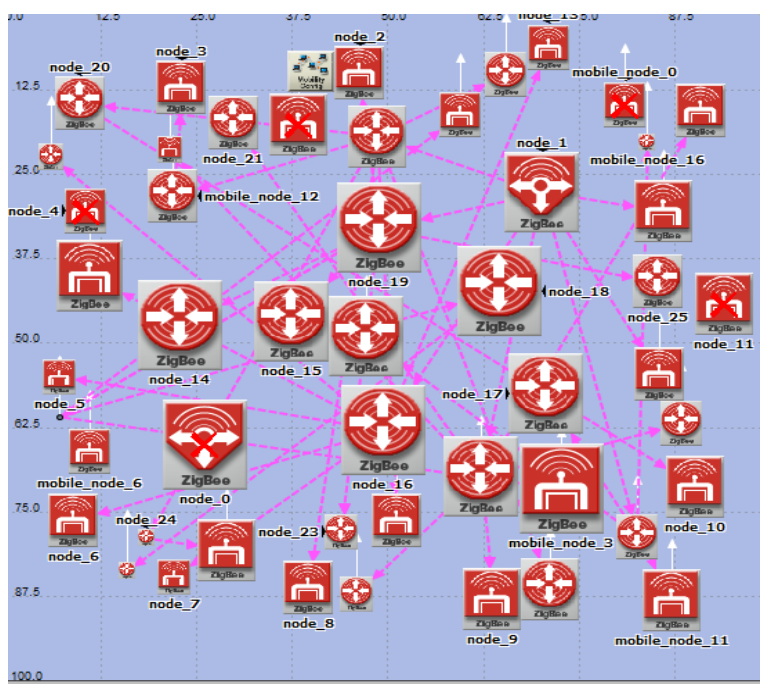

Fig 1: MT network with failed devices 


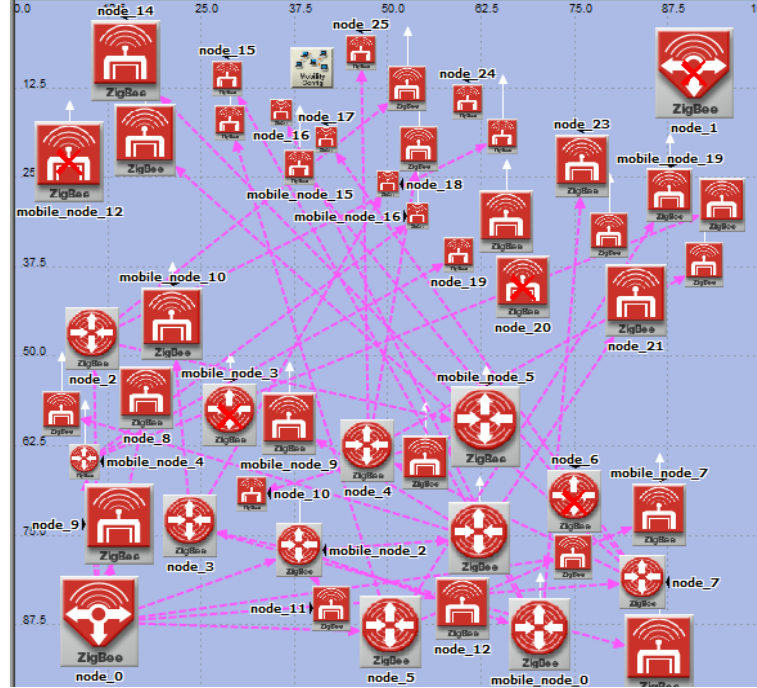

Fig 2: SM network with failed devices

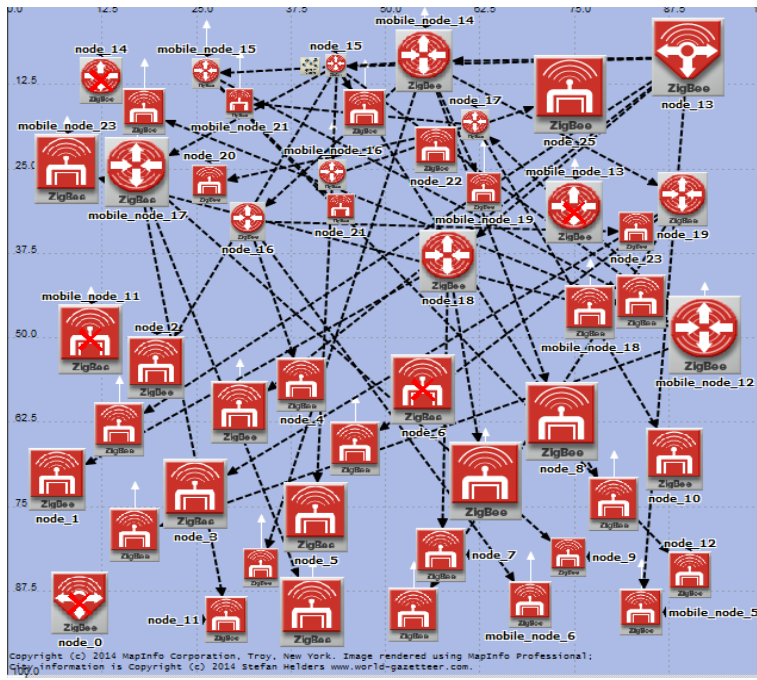

Fig 3: ST network with failed devices

Whereas figure 1,2 and 3 shows the hybrid MT, SM and ST networks. Here in the given figures it will be clearly shown that if one coordinator will be failed than another coordinator will overtake the network. While in case single coordinator network if the coordinator failed then whole network will be failed and stop working.

\section{RESULTS and DISCUSSIONS}

On the basis of above assumptions following result have been observed:

\subsection{Throughput}

Throughput is the ratio of the total amount of data that a receiver receives from a sender to a time it takes for receiver to get the last packet. Throughput is the data quantity transmitted correctly starting from the source to the destination within a specified time (seconds). A low delay in the network translates into higher throughput. Throughput is quantified with varied factors including packet collisions, obstructions between nodes and the type of used topology.

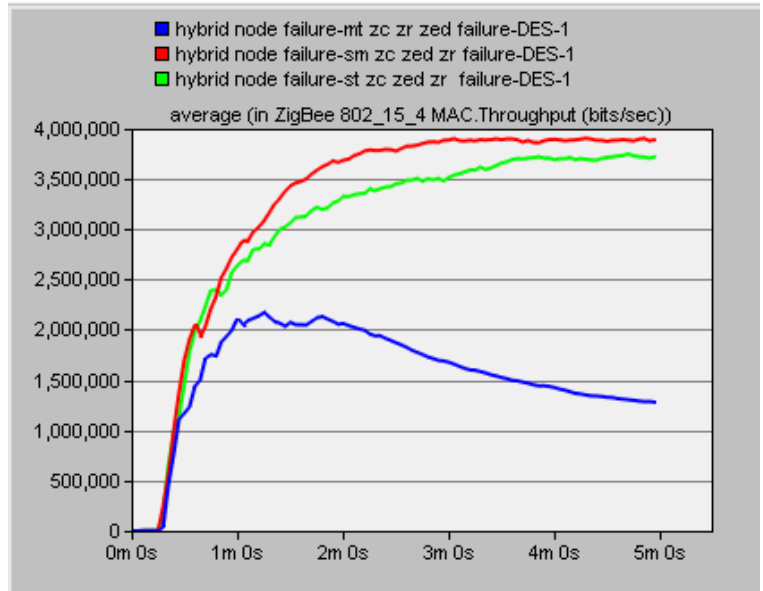

Fig 4: Throughput of Hybrid Topologies in Case of Device Failure

Maximum throughput as shown in fig. 4 achieves by SM network but there is less difference between the throughput of SM and ST, whereas minimum throughput achieve by MT network.

\subsection{Data Dropped:}

This statistic records the total amount of data that was received from the upper layer and then dropped by all nodes in the network due to repeatedly failed retransmissions (i.e., exceeded the corresponding short retry or long retry threshold value).

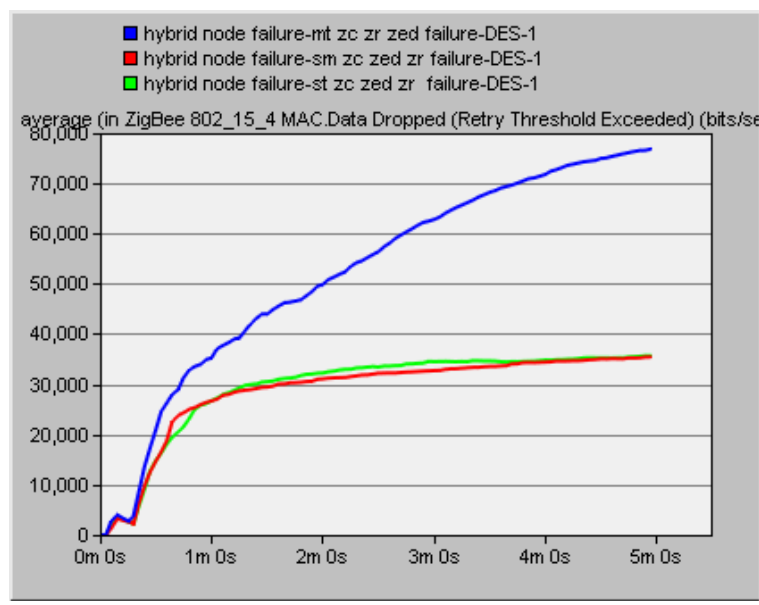

Fig 5: Data Dropped by Hybrid Topologies in Case of Device Failure

Maximum data will be discharged by MT network at the MAC layer where as SM and ST dropped minimum data at the MAC layer as referred to fig5.

\subsection{End to End Delay (E2ED)}

End-to-End Delay refers to the time taken for a packet to be transmitted across a network from source to destination.

$$
\begin{aligned}
& \mathrm{d}_{\text {end-end }}=\mathrm{N}\left[\mathrm{d}_{\text {trans }}+\mathrm{d}_{\text {prop }}+\mathrm{d}_{\text {proc }}\right] \\
& \text { where, } \\
& \mathrm{d}_{\text {end-end }}=\text { end-to-end delay } \\
& \mathrm{d}_{\text {trans }}=\text { transmission delay } \\
& \mathrm{d}_{\text {prop }}=\text { propagation delay } \\
& \mathrm{d}_{\text {proc }}=\text { processing delay }
\end{aligned}
$$


$\mathrm{N}=$ number of links (Number of routers +1 ). Each router will have its own $d_{\text {trans }}, d_{\text {prop}}, d_{\text {proc }}$ hence this formula gives a rough estimate.

The result shown in Fig. 6 described that minimum delay achieved by ST and SM networks.

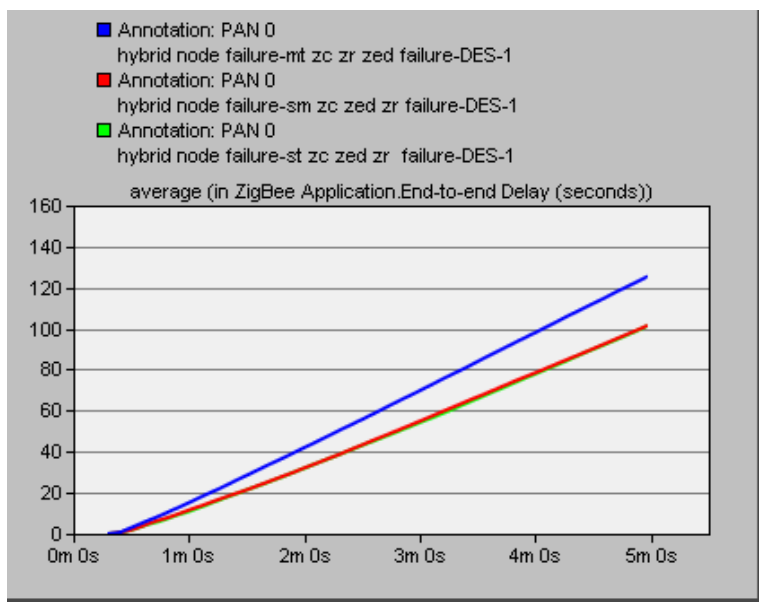

Fig 6: E2ED of Hybrid Topologies in Case of Device Failure

\subsection{Packet Dropped}

This statistic show the packet not joined at the Network Layer. The hybrid ST network dropped minimum number of packet (which contains data) at the network layer. In case of MT network the maximum Packet should be dropped at the Network layer as shown in fig. 7.

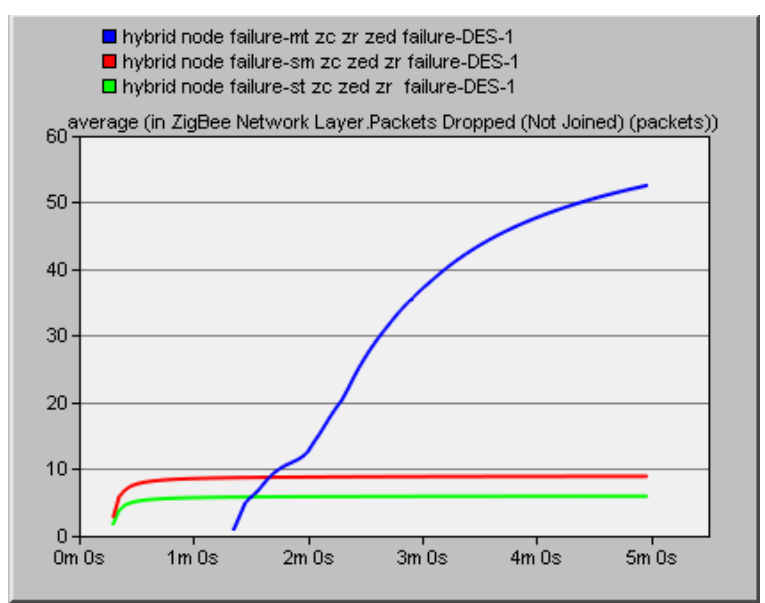

Fig 7: Packet Dropped by Hybrid Topologies in Case of Device Failure

\subsection{Network Load}

This statistic records the total amount of data submitted by the upper layer for transmission by the physical layer on all the nodes in the network. The maximum network load will be discovered by the coordinator of MT network as referred to fig. 8 , which lead to the maximum usage of energy.

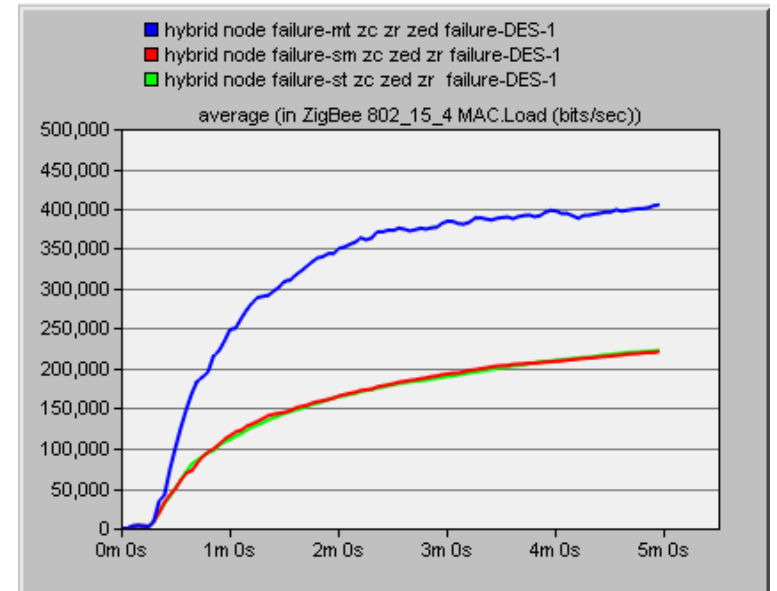

Fig 8: Network Load on Hybrid Topologies in Case of Device Failure

\subsection{Data Traffic Received (DTR)}

These statistics record successfully received data traffic on this network interface from the physical layer. When these statistics are reported in units of bits/second, the physical and the MAC header sizes are included in the computation of the total amount of traffic received. These statistics record all the data received on the network interface regardless of the destination address. The result shown in fig. 9 given below shows that maximum DTR should be discovered by SM and minimum in case of MT network.

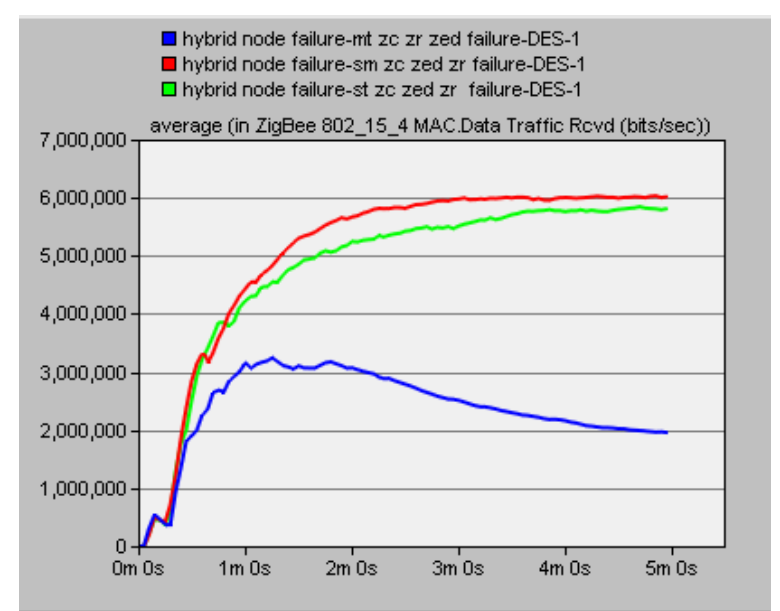

Fig 9: DTR in Hybrid Topologies in Case of Device Failure

\subsection{Data Traffic Sent (DTS)}

These statistics record the amount of data transmitted by the network interface onto the physical layer. When these statistics are reported in units of bits/second, the physical and the MAC header sizes are included in the computation of the total amount of traffic sent. The result shown in fig. 10 given below shows that maximum DTS should be discovered by MT and minimum in case of SM and ST network. 


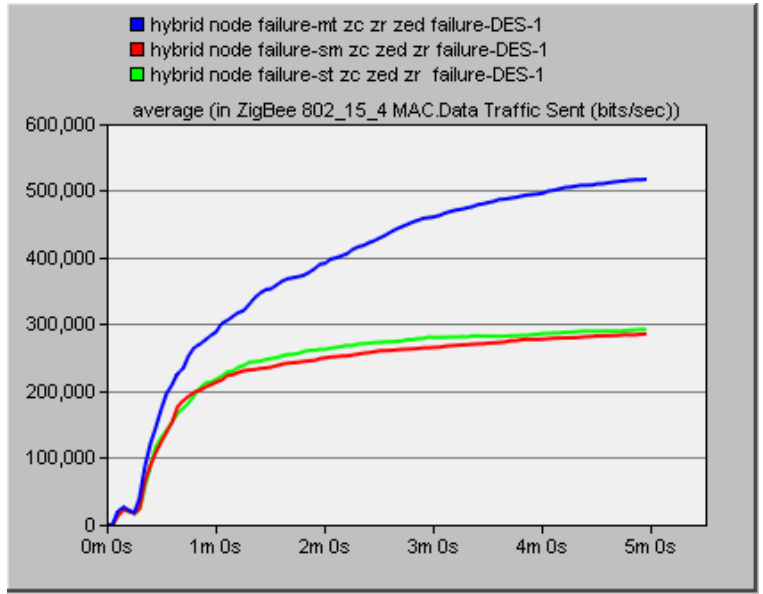

Fig 10: DTS in Hybrid Topologies in Case of Device Failure

\subsection{Number of Hops}

It is the average number of hops traveled by application traffic in the PAN. The hop count represents the total number of devices a given piece of data (packet) passes through i.e. the intermediate devices (like routers) through which data must pass between source and destination. The number of hops shown in fig. 11 is genuine for all networks and within the prescribed limits.

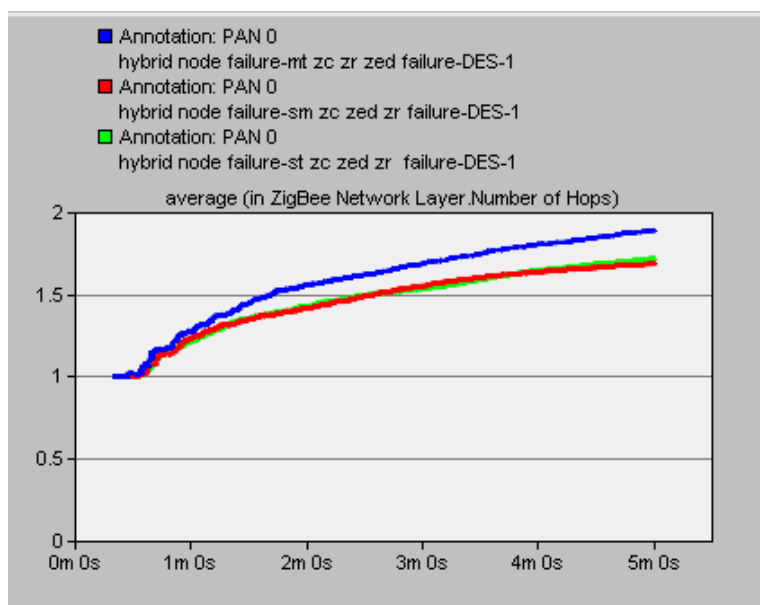

Fig 11: Number of Hops in Hybrid Topologies in Case of Device Failure

\section{CONCLUSION}

This research work deals with the performance of hybrid topologies and their performance compared on basis of device malfunctioning. The above performance metrics shows that maximum throughput achieves by SM network but there is less difference between the throughput of SM and ST, whereas minimum throughput achieve by MT network. The maximum DTS should be discovered by MT and minimum in case of SM and ST network and the maximum DTR should be discovered by SM and minimum in case of MT network. The maximum network load will be discovered by the coordinator of MT network. The hybrid ST network dropped minimum number of packet at the network layer. In case of MT network the maximum Packet should be dropped at the Network layer and maximum data will be discharged by MT network at the MAC layer where as SM and ST dropped minimum data at the MAC layer. ST and SM networks achieved minimum delay as compared to MT network. According to above discussed results, to design an effective network whose performance is excellent it is essential to assign star topology to one of network coordinator and another one should be mesh or tree it doesn't affect the network in case of device failure, because the SM and ST network almost gives the similar results.

\section{REFFERENCES}

[1] Karandeep Malhi, Subhas Chandra Mukhopadhyay, MathiasHaefke, and Hartmut Ewald, "A Zigbee - Based Wearable Physiological Parameterz Monitoring System", IEEE sensor journal, vol. 12 NO. 3, march 2012.

[2] Leonardo Goratti, Jussi Haapola, "Highly Reliable Star and Sub-Mesh Hybrid Sensor Network for Smart Grid Monitoring" GC'12 Workshop: Smart Grid Communications: Design for Performance, IEEE GLOBCOM workshop 2012:1480-1485.

[3] Dong Wook Seo, Doo Seop Yun, Sung Ho Cho, "A Network Performance Improvement for One-to-One and Many-to-One Communication Environment in Zigbee" 2007 International Conference on Applied Electronics (CD-ROM), Plzen, Czech Republic, September 5-7, 2007.

[4] Francesca Cuomo, Emanuele Cipollone, Anna Abbagnale"Performance analysis of IEEE 802.15.4 wireless sensor networks: An insight into the topology formation process" ELSEVIER, Computer Networks 53 (2009) 3057-3075.

[5] J. Pedro Amaro, Fernando J.T.E., Ferreira,RuiCortesão, Jorge Landeck, "Powering Wireless Sensor Networks Nodes for Comple Protocols on Harvested Energy", CENTERIS 2012 - Conference on Enterprise Information Systems HCIST 2012 - International Conference on Health and Social Care Technologies, Procedia Technology 5 ( 2012 ) 518 - 526.

[6] Joa-Hyoung Lee, In-Bum Jung, "Speedy Routing Recovery Protocol for Large Failure Tolerance in Wireless Sensor Networks", SENSORS ISSN 14248280 .

[7] Mumtaj M.Ali Al-Mukthar, Teeb Hussain Hadi, "Modeling the Performance of Zigbee Cluster Tree Wireless Sensor Network in Presence of Failure", Journal of Advanced Computer Science and Technology Research,Vol.3 No.3, September 2013, 116-126.

[8] Macro Gribaudo, Daniele Manini, Alessandro Nordio "trnsient analysis of IEEE 802.15.4 sensor network" IEEE Transaction on Wireless communication VOL. 10 No. 4 April 2011.

[9] Jing Sun, Xiaofen Zhang, "Study of ZigBee Wireless Mesh Networks" 2009 Ninth International Conference on Hybrid Intelligent Information Systems and Systems, vol.02, IEEE Computer Society Washington,DC,USA @ 2009.

[10] Jun Wang, Victor C. M. Leung, "Comparisons of Home Area Network Connection Alternatives for Multifamily Dwelling Units" $20114^{\text {th }}$ IFIP International Confrence on New Technologies, Mobility and security NTMS 2011 in paris (france), C)2011 IEEE.

[11] Bogdan Pavkovic, Andrzej Duda, Won-Joo Hwang, Fabrice Theoleyre, "Efficient topology construction for RPL over IEEE 802.15.4 in wireless sensor network" ELSEVIER Ad-hoc networks. 